\title{
Erratum to: Corrosion Behavior of Electroless Nickel/Immersion Gold Plating by Interfacial Morphology
}

\author{
Authors: Dong-Jun Lee, Seok-Hwan Huh, Chi-Seong Kim, Seongjae Mun, \\ Han-Kyun Shin, and Hyo-Jong Lee
}

DOI: $10.1007 / \mathrm{s} 13391-015-4446-\mathrm{x}$

The acknowledgement in the first online article has been corrected as given in the regular issue. 